\title{
Tendances d'évolution des peuplements de poissons de la Seine en réponse à la variabilité hydroclimatique
}

\section{Temporal evolution of fish communities in the Seine river in response to the hydroclimatic variability}

\author{
É. Tales \\ Cemagref, Unité de recherche Hydrosystèmes et bioprocédés, BP 44, Parc de Tourvoie, \\ 92163 Antony Cedex, France \\ e-mail : evelyne.tales@cemagref.fr
}

Résumé - L'objectif de cette étude est d'analyser l'évolution temporelle récente des peuplements de poissons de la Seine en liaison avec l'évolution des conditions hydroclimatiques (i.e. variations de la température de l'eau et du débit). Cette évolution temporelle est examinée selon deux voies complémentaires : la variabilité inter-annuelle du recrutement et l'évolution des peuplements de poissons sur l'axe Seine. Des analyses de co-inertie permettent de rechercher les relations entre les peuplements de poissons et les mesures de température et de débit. Le recrutement, étudié par le biais des jeunes poissons de l'année dans un site de la Seine entre 1999 et 2004, varie seulement en réponse aux variations des extrêmes du régime thermique (les températures hautes durant l'année biologique et les températures basses durant la période de reproduction), les moyennes thermiques n'étant pas significatives. En revanche, les peuplements de poissons adultes recensés dans deux stations de l'axe Seine entre 1990 et 2004 ne présentent pas de forte corrélation avec les conditions hydroclimatiques.

Mots clés - peuplements de poissons, variation temporelle, Seine, température, recrutement

\begin{abstract}
Analysing the recent temporal evolution of fish assemblages of the Seine River with respect to variations of water temperature and discharge is the main aim of this study. Two analyses were performed, concerning successively inter-annual variations of recruitment and temporal evolution of fish assemblages of the main Seine River. Relationships between fish and water temperature and discharge data were assessed using co-inertia analysis. The recruitment, studied in one sampling site between 1999 and 2004, varies only in relation to extreme values in the thermal regime (higher water temperatures of biological year and lower water temperatures of reproduction season). Mean water temperatures have no significant effect on recruitment variations. In contrast, there is no correlation at all between adult fish assemblages of the main Seine River sampled between 1990 and 2004 in two study sites, and hydroclimatic conditions.
\end{abstract}

Key words - fish assemblages, temporal evolution, Seine River, temperature, recruitment 


\section{INTRODUCTION}

Il est maintenant avéré que la température du globe augmente progressivement, influencée par les activités humaines, entraînant un changement climatique global. Ce changement va obligatoirement avoir des effets sur les organismes vivants et il est important de les connaître pour en estimer les conséquences. L'objectif est donc d'analyser l'évolution temporelle des peuplements biologiques sous contrainte du changement climatique.

Le facteur thermique est particulièrement important pour les organismes poïkilothermes tels les organismes aquatiques de part leur écologie. De plus, ils sont soumis à l'influence spécifique d'activités humaines générant le rejet d'effluents thermiques comme les installations de production d'électricité. Dans ce contexte, il faut pouvoir déterminer les effets respectifs de l'augmentation de la température de l'eau sur les organismes aquatiques, liée d'une part au réchauffement global et d'autre part aux rejets thermiques des centrales de production d'électricité.

Cette étude comporte deux démarches pour appréhender l'évolution temporelle des peuplements de poissons de la Seine, en réponse à la variabilité hydroclimatique :

- l'étude de la variation inter-annuelle du recrutement, qui permet d'analyser la réponse biologique «en temps réel » à cette variabilité, la phase de reproduction étant directement liée aux amplitudes thermiques saisonnières ;

- l'étude des tendances d'évolution à plus long terme par la comparaison conjointe des peuplements piscicoles de 2 stations situées sur l'axe Seine durant près de 15 ans.

Ainsi, ces deux approches sont complémentaires pour évaluer l'effet de la variabilité hydroclimatique sur la faune piscicole des cours d'eau.

\section{MATÉRIELS ET MÉTHODES}

\subsection{Présentation des stations}

Trois sites sont étudiés, qui se succèdent le long de la Seine, en amont de Paris (Fig. 1A). Le premier site correspond au Centre Nucléaire de Production d'Électricité (CNPE) de Nogent-sur-Seine (Aube). Le site 2 se situe à proximité de Bray-sur-Seine (Seine-et-Marne), en aval du barrageécluse de la Grande Bosse, à $45 \mathrm{~km}$ à l'aval du CNPE de Nogent-sur-Seine. Le dernier site est à Choisy-le-Roi (Val-de-Marne), à environ $150 \mathrm{~km}$ en aval du CNPE de Nogent-sur-Seine. Situés en amont de Paris, ils ne subissent donc pas l'influence des rejets de la capitale. Le site de Choisy-leRoi est toutefois en zone urbanisée, et plus fortement anthropisé que ceux de Nogent-sur-Seine et de Bray-surSeine.

Le recrutement a été étudié exclusivement dans le site 2. Ce site comporte deux stations qui ont fait l'objet d'un suivi durant 6 années (1999-2004) dans le cadre d'un projet expérimental pour réaliser une opération de réhabilitation (Fig. 1B). Ces deux stations, le bras de la Grande Bosse et le bras de la Boule, sont d'anciens méandres de la Seine, rescindés lors de l'aménagement pour les besoins de la navigation. 


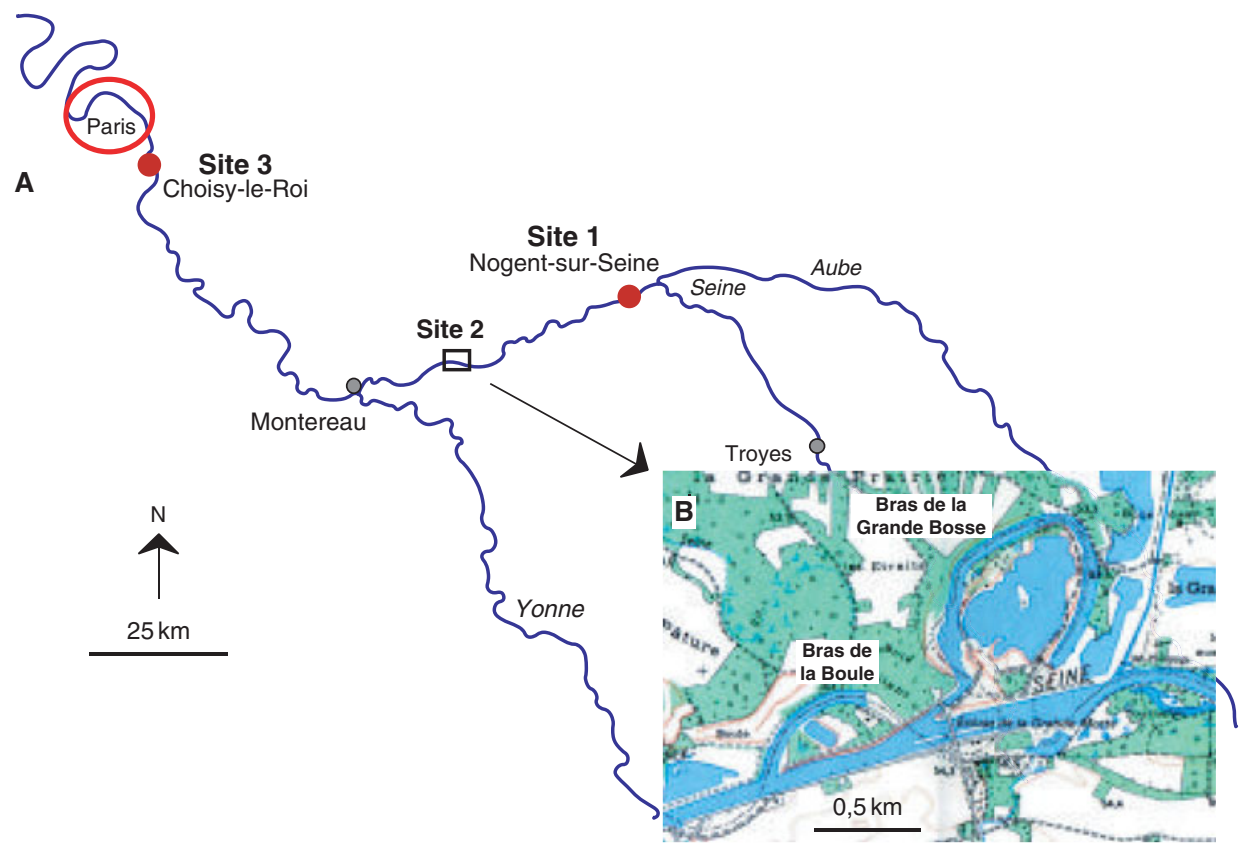

Fig. 1. A) Localisation des trois sites d'étude dans le bassin de la Seine, B) détails des stations du site 2 (source : d'après la carte IGN 1/25000 n 2516 est).

Fig. 1. A) Location of the three study sites in the Seine basin; B) Stations of site 2 (after IGN map $\mathrm{n}^{\circ} 2516$ East).

Le bras mort de la Grande Bosse, d'une longueur totale de $1,7 \mathrm{~km}$ et d'une profondeur n'excédant pas les 3 mètres, n'a jamais été navigué. II est en communication avec la Seine par son extrémité aval. Son état de comblement est assez avancé d'où une faible profondeur par endroits et surtout en amont. Le bras de la Boule, situé plus en aval, est de taille nettement inférieure à celle du précédent. Sa longueur est de $600 \mathrm{~m}$ et sa profondeur maximale de $4 \mathrm{~m}$.

Ces stations sont modérément soumises aux variations hydrologiques puisque ce sont donc des bras morts en connexion permanente avec le chenal principal qui est navigué. Cette caractéristique devrait permettre d'analyser spécifiquement l'influence du facteur thermique sur les peuplements de jeunes poissons dans ces stations.

L'évolution à long terme des peuplements de poissons de l'axe Seine a été plus particulièrement étudiée dans les sites 1 et 3 (Fig. 1A). Concernant le site du CNPE de Nogent-surSeine, la station retenue se situe à l'aplomb de la centrale : en effet, à cette station précisément, la Seine est localement aménagée (berges homogènes en enrochements) et subit également l'effet régulateur d'un barrage 
de navigation situé immédiatement à l'aval. Ces caractéristiques la rapprochent morphologiquement de la station de Choisy-le-Roi, et contribuent à rendre comparables ces deux sites même si leur contexte est différent. Bien entendu, aucun des sites n'a subi de modifications physiques durant la période étudiée.

\subsection{Données piscicoles}

Les méthodes d'échantillonnage des peuplements de poissons diffèrent selon les objectifs de l'étude. Pour l'étude concernant les variations du recrutement, l'échantillonnage a concerné spécifiquement les jeunes poissons de l'année dans le site 2 . Les prélèvements ont été effectués par pêche électrique selon la méthode des EPA (échantillonnage ponctuel d'abondance), depuis un bateau pneumatique, au moyen d'un appareil portable de pêche électrique (modèle Martin Pêcheur). Cet appareil est équipé d'une anode circulaire de taille réduite (diamètre de $10 \mathrm{~cm}$ ) pour renforcer son efficacité vis-à-vis des poissons de petite taille. Les points de pêche sont répartis aléatoirement. Les poissons capturés sont identifiés et mesurés au millimètre près sur place, et remis immédiatement à l'eau. Les individus non identifiables sur le terrain sont conservés dans du formol à $5 \%$ et déterminés ultérieurement au laboratoire à la loupe binoculaire. Une analyse de la distribution de fréquence de taille des individus de chaque espèce permet de sélectionner les jeunes poissons de l'année parmi tous les poissons capturés.

Chaque année du suivi comporte trois campagnes de prélèvement réali- sées à la saison estivale (fin juin, fin juillet et début septembre). Le nombre de points réalisés par station, relatif à leur taille, est de 120 par campagne (bras de la Grande Bosse : 80 points; bras de la Boule : 40 points). Ce suivi a été réalisé de 1999 à 2004.

Pour étudier plus globalement l'évolution des peuplements de poissons sur l'axe Seine, dans les sites 1 et 3, c'est l'ensemble du peuplement qui a été pris en compte. L'échantillonnage de ces deux sites est réalisé une fois par an, au printemps, par pêche électrique depuis un bateau (en continu, par ambiance ou par échantillonnage ponctuel d'abondance). Le site de Nogent-sur-Seine est suivi depuis 1987 dans le cadre du suivi hydrobiologique du CNPE. Le site de Choisy-le-Roi fait partie du Réseau Hydrobiologique et Piscicole (RHP) qui constitue un des réseaux de surveillance de la qualité des cours d'eau. Ce site n'est suivi que depuis 1990 par le Conseil Supérieur de la Pêche (CSP, actuellement ONEMA). Par conséquent, les données retenues concernent la période commune aux deux sites, de 1990 à 2004.

\subsection{Données environnementales}

Les données de température utilisées sont toutes des mesures directes de température de l'eau provenant de diverses origines. Pour le site 1, les mesures proviennent des suivis en continu réalisés par EDF à l'amont du CNPE. EDF (DTG) a fourni les moyennes de température de l'eau calculées sur trois périodes annuelles : du $1^{\text {er }}$ juillet au 31 octobre, du $1^{\text {er }}$ avril au 30 juin, et sur l'ensemble de l'année biologique (du $1^{\mathrm{er}}$ 
juillet au 30 juin). Ces périodes sont définies en raison de leur pertinence relative au cycle de vie des espèces de poissons. En effet, l'année biologique définie ici intègre le cycle de vie des espèces cyprinicoles en particulier, qui débute au printemps. La période du $1^{\text {er }}$ juillet au 31 octobre (donc le début de l'année biologique), période estivale, correspond au stade de développement des jeunes poissons de l'année $(0+)$. La période du $1^{\text {er }}$ avril au 30 juin (la fin de l'année biologique), période printanière, correspond quant à elle à la période de reproduction de la plupart des espèces piscicoles de ce secteur de la Seine. Dans le site 3, à Choisy-leRoi, les données disponibles sont des moyennes journalières, fournies par Veolia qui effectue des mesures de température de l'eau de la Seine à l'aplomb de la prise d'eau de sa station d'eau potable. Pour homogénéiser les données thermiques des sites 1 et 3 , les calculs des moyennes de température aux trois périodes annuelles précédemment évoquées ont été réalisés pour le site 3 .

Les données thermiques du site 2 , destinées à l'étude de la variabilité du recrutement, sont issues d'enregistreurs de température, autonomes et étanches (modèle StowAway de marque Prosensor), disposés dans chacune des deux stations. Ils ont été configurés pour effectuer une mesure horaire de la température de l'eau et ont été relevés plusieurs fois par an. À partir de ces mesures horaires, la température moyenne journalière est calculée. Sur toute la durée du suivi, il existe des lacunes ponctuelles dans le jeu de données brutes suite à des problèmes techniques. Dans ce cas-là, les données manquantes ont été reconstituées par interpolation : en effet, en dépit de ces problèmes, nous avons toujours disposé d'au moins une série de données complète. Bien qu'il existe une légère variabilité spatiale entre les régimes thermiques des deux stations, les températures aux deux stations sont extrêmement corrélées $\left(R^{2}=0,976 ; p<0,0001\right)$, ce qui justifie la constitution de séries complètes par interpolation.

Des données hydrométriques sont également nécessaires pour analyser l'évolution à long terme des peuplements de poissons de la Seine dans les sites 1 et 3 . Deux stations de jaugeage du réseau de la banque HYDRO sont proches des deux sites d'étude (Pont-sur-Seine en amont proche de Nogent-sur-Seine, et Alfortville à l'aval immédiat de Choisyle-Roi), et ont fourni les mesures journalières de débit. Afin de rendre l'ensemble des données mésologiques comparables, les moyennes de débit ont été calculées pour les deux sites, de manière analogue à celles des températures de l'eau : sur chaque année biologique (du $1^{\text {er }}$ juillet au 30 juin), sur la période estivale de chaque année biologique (du $1^{\text {er }}$ juillet au 31 octobre), et sur la période printanière ( $1^{\mathrm{er}}$ avril au 30 juin). Ceci permet d'avoir les caractéristiques de débits et de températures de l'eau sur les mêmes périodes pour les deux stations étudiées.

\subsection{Analyses}

Les analyses effectuées prennent en compte deux types de variables, les variables biologiques et les variables de milieu. Les variables biologiques prises en compte correspondent aux 
abondances des espèces capturées chaque année dans les différents sites. Les espèces minoritaires (occurrence inférieure à 2 dans l'ensemble du suivi) sont écartées au préalable du jeu de données analysé. Les abondances des autres espèces, ramenées à un même effort de pêche (Captures Par Unité d'Effort de pêche, CPUE, de 15 minutes), sont transformées en $\log (x+1)$ pour stabiliser leur variance. II y a donc autant de variables biologiques que d'espèces capturées. Les variables de milieu, thermiques et hydrométriques, sont calculées à partir des mesures journalières disponibles. Pour l'étude de l'évolution à long terme des peuplements de poissons de l'axe Seine, ces variables correspondent aux moyennes calculées sur les trois périodes annuelles (année biologique entière, période estivale et période printanière) et sont au nombre de six (trois moyennes thermiques et trois moyennes de débit). La démarche est un peu différente pour l'étude de la variabilité du recrutement en réponse aux conditions hydroclimatiques. Tout d'abord, le débit n'est pas pris en compte puisque les stations d'étude sont des milieux strictement lentiques. Par ailleurs, les variables thermiques utilisées sont issues d'une analyse des centiles des températures journalières, telle que définie par Cattaneo et al. (2002). Les centiles 10 et 90 (variables T10 et T90) permettent de caractériser les températures extrêmes observées, soit l'amplitude de variation des températures; le centile 50 (i.e. la médiane) (variable T50) caractérise la tendance thermique moyenne et le coefficient de variation (variable CV) permet d'estimer la variabilité thermique. Ces 4 paramètres ont été calculés sur deux périodes : l'année biologique «biol » (1 an du $1^{\text {er }}$ juillet année n-1 au 30 juin année $n$ ) et la période «repro» de reproduction et croissance des jeunes poissons de la plupart des espèces présentes dans les stations étudiées (Cyprinidae) (du $1^{\text {er }}$ avril au 30 septembre de l'année $n$ ). Ainsi, les variables thermiques sont au nombre de huit (4 variables sur 2 périodes) dans l'étude de la variabilité du recrutement.

Les analyses effectuées consistent à rechercher les relations entre les variables biologiques et les variables de milieu et sont déduites d'une analyse de co-inertie. Cette analyse consiste en un couplage symétrique de deux tableaux de données, le tableau des variables de milieu et le tableau faunistique, chacun des tableaux étant traité au préalable par une analyse en composantes principales (ACP). Ce type d'analyse recherche une costructure commune aux deux matrices de données selon le critère d'optimisation de la covariance. Cette méthode s'avère par ailleurs extrêmement robuste quand le nombre d'observations est faible relativement aux nombres de variables et de taxons considérés (Dolédec \& Chessel, 1994), ce qui est souvent le cas en écologie. L'analyse est réalisée à l'aide du module Co-inertia de ADE.

\section{RÉSULTATS}

\subsection{Réponse du recrutement à la variabilité thermique inter-annuelle (1999-2004)}

\section{Variabilité de la température}

En raison de la forte corrélation et synchronie des températures 


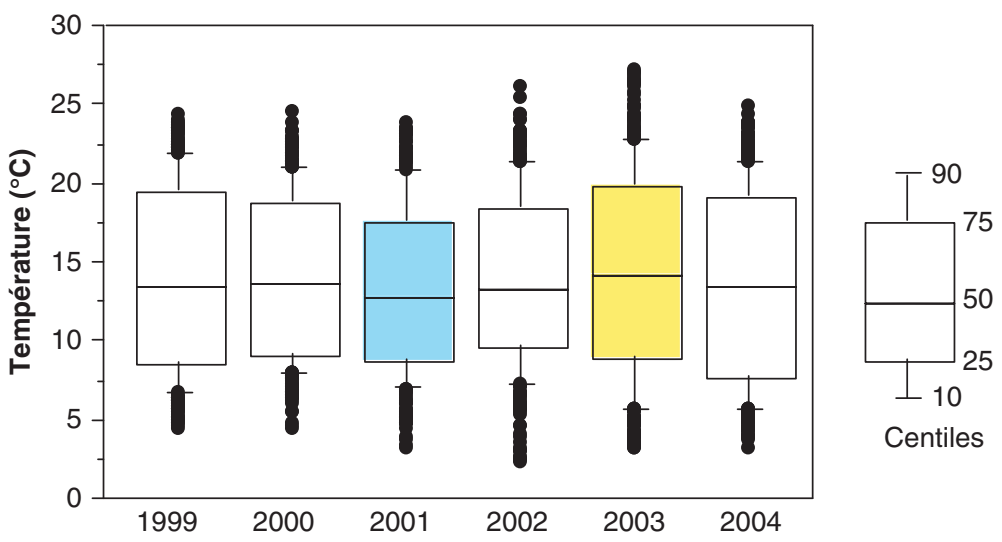

Fig. 2. Évolution annuelle des températures à la station de la Grande Bosse. Les «boîtes» comprennent les valeurs entre les percentiles 25 et 75, la médiane étant indiquée à l'intérieur des boîtes. Les «moustaches» correspondent aux percentiles 10 et 90 . Les points correspondent enfin aux valeurs extrêmes.

Fig. 2. Annual evolution of water temperature at station "Grande Bosse". The boxes include values between percentiles 25th and 75th. Median value is indicated into the boxes. "Wiskars" correspond to percentiles 10th and 90th. Black points are extreme values.

aux deux stations du site 2, l'analyse préliminaire de leur variabilité a été restreinte aux données de température de l'eau du bras de la Grande Bosse. II n'y a pas de tendance d'évolution particulière qui se dégage sur la période étudiée. Sur la durée du suivi, l'année 2001 est la plus « fraîche » (Température moyenne annuelle : $13,38^{\circ} \mathrm{C}$ et médiane : $12,81^{\circ} \mathrm{C}$ ) tandis que l'année 2003 est la plus chaude (température moyenne annuelle : $14,25^{\circ} \mathrm{C}$ et médiane : $14,21^{\circ} \mathrm{C}$ ) (Fig. 2).

L'année 2003 se caractérise également par une large amplitude de variation de la température, l'épisode de canicule ayant entraîné l'occurrence de températures exceptionnellement fortes en période estivale.

La visualisation de l'évolution annuelle de la température lors de ces
2 années contrastées, 2001 et 2003, met en évidence que les écarts de température sont saisonniers (Fig. 3). Notamment, c'est surtout au printemps et en été que les écarts sont les plus marqués, ce qui correspond à la période de reproduction et de croissance de la plupart des espèces de poissons recensées dans ce secteur de la Seine.

Variabilité des peuplements de jeunes poissons de l'année

Pour les 6 années considérées, 5154 individus ont été capturés aux deux stations, appartenant à 22 espèces (Tab. I). Les espèces dominantes en termes d'effectifs sont le gardon (2525 individus) suivi par la perche commune (681 individus), l'ablette (606 individus) et le chevesne 


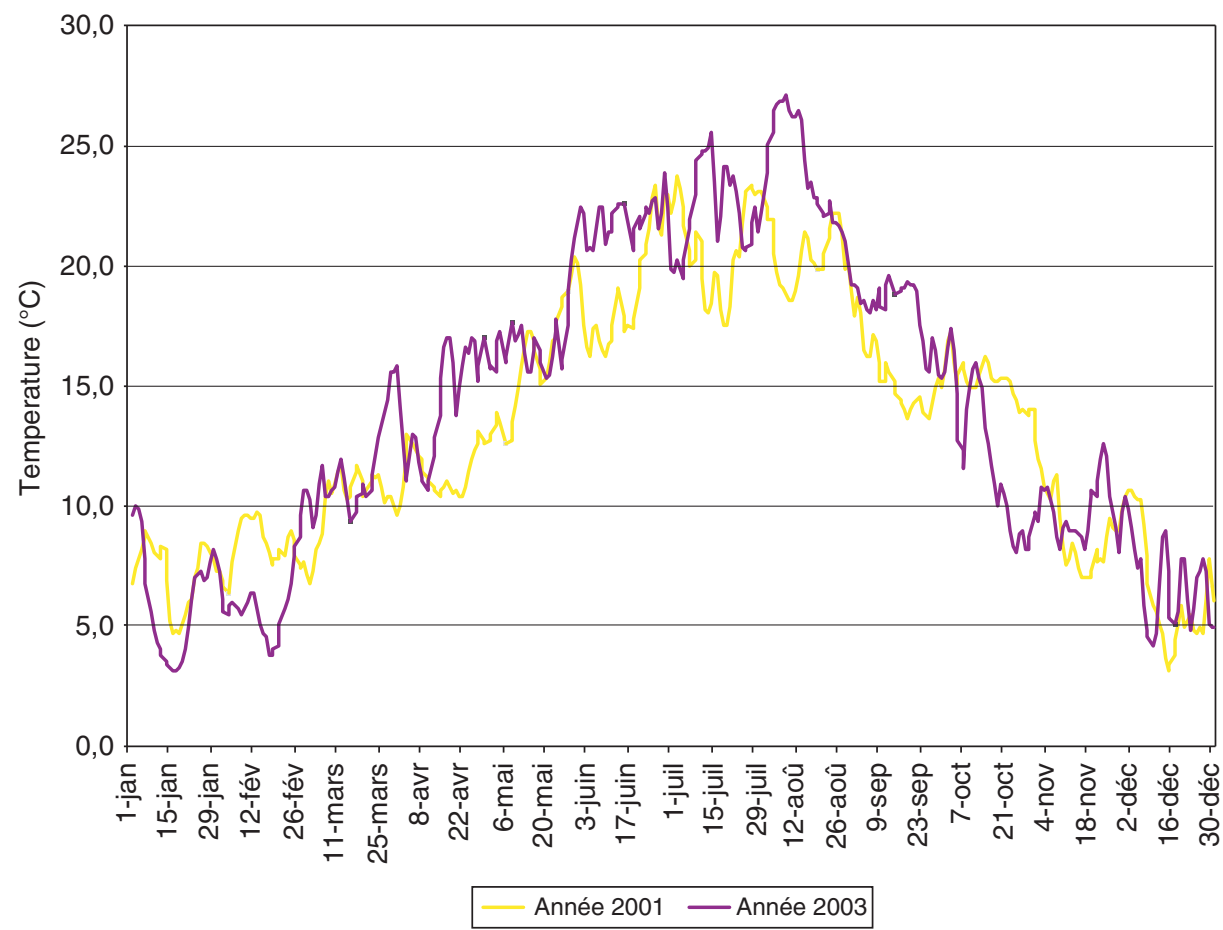

Fig. 3. Évolution annuelle de la température moyenne journalière de l'eau à la station de La Grande Bosse en 2001 et 2003.

Fig. 3. Annual evolution of mean daily water temperature at station "Grande Bosse" in 2001 and 2003.

(402 individus) (Tab. I). À l'inverse, six espèces ont des effectifs totaux inférieurs à 5 (carpe commune, épinoche, loche de rivière, poisson chat, sandre et silure).

Globalement, la richesse spécifique totale, comprise entre 11 et 16 espèces selon les années, a augmenté au cours du suivi (Fig. 4). Cette augmentation est essentiellement liée à celle de la richesse spécifique observée à la station Grande Bosse, la richesse spécifique de la station La Boule ayant plutôt tendance à être stable. Elle est causée par l'apparition des espèces « minoritaires » (loches, sandre, silure) dans les captures au cours des dernières années. En revanche, l'équitabilité diminue entre 1999 et 2004 (Fig. 5).

Une étude antérieure (Caudy, 2004) a montré qu'il existe une évolution temporelle des jeunes poissons de l'année très similaire entre les deux stations considérées, en dépit de certaines spécificités faunistiques respectives : par exemple, la présence du rotengle et de la perche soleil est caractéristique de la station La Boule 
Tableau I. Liste des 22 espèces capturées et de leurs effectifs dans le site 2 entre 1999 et 2004.

Table I. List of 22 caught species and their numbers in the site 2 between 1999 and 2004.

\begin{tabular}{|c|c|c|c|c|}
\hline \multirow{2}{*}{$\begin{array}{l}\text { Famille } \\
\text { Esocidae }\end{array}$} & \multicolumn{2}{|c|}{ Espèce } & \multirow{2}{*}{$\frac{\text { Code espèce }}{\text { BRO }}$} & \multirow{2}{*}{$\frac{\text { Effectifs totaux bruts }}{13}$} \\
\hline & Esox lucius & brochet & & \\
\hline \multirow[t]{13}{*}{ Cyprinidae } & Abramis brama & brème & BRE & 213 \\
\hline & Alburnus alburnus & ablette & $A B L$ & 606 \\
\hline & Barbus barbus & barbeau & BAF & 11 \\
\hline & Blicca bjoerkna & brème bordelière & BRB & 236 \\
\hline & Chondrostoma nasus & hotu & HOT & 14 \\
\hline & Cyprinus carpio & carpe commune & $\mathrm{CCO}$ & 1 \\
\hline & Gobio gobio & goujon & GOU & 121 \\
\hline & Leuciscus cephalus & chevesne & $\mathrm{CHE}$ & 402 \\
\hline & Leuciscus leuciscus & vandoise & VAN & 9 \\
\hline & Rhodeus sericeus & bouvière & $\mathrm{BOU}$ & 155 \\
\hline & Rutilus rutilus & gardon & GAR & 2525 \\
\hline & Scardinius erythrophthalmus & rotengle & ROT & 52 \\
\hline & Tinca tinca & tanche & TAN & 25 \\
\hline Cobitidae & Cobitis taenia & Loche de rivière & LOR & 3 \\
\hline Balitoridae & Barbatula barbatula & loche franche & LOF & 19 \\
\hline lctaluridae & Ameiurus melas & poisson-chat & $\mathrm{PCH}$ & 3 \\
\hline Gasterosteidae & Gaterosteus aculeatus & épinoche & EPI & 1 \\
\hline Siluridae & Silurus glanis & silure & SIL & 1 \\
\hline \multirow[t]{2}{*}{ Percidae } & Perca fluviatilis & perche & PER & 681 \\
\hline & Stizostedion lucioperca & sandre & SAN & 4 \\
\hline Centrarchidae & Lepomis gibbosus & perche-soleil & PES & 58 \\
\hline
\end{tabular}

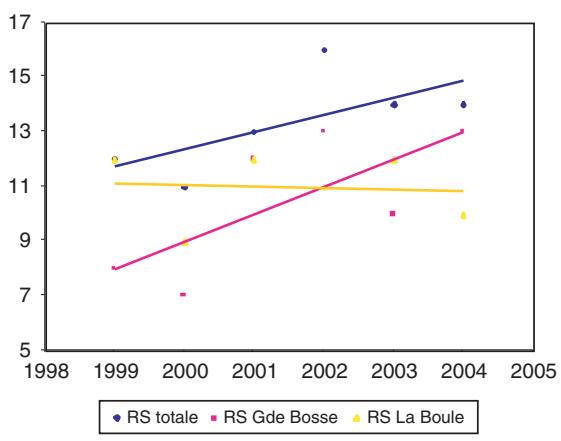

Fig. 4. Évolution de la richesse spécifique aux deux stations de 1999 à 2004.

Fig. 4. Evolution of species richness in the two stations between 1999 and 2004.

alors que la bouvière et la brème commune le sont pour la station Grande Bosse.

Ainsi, nous nous focalisons directement par la suite sur les relations

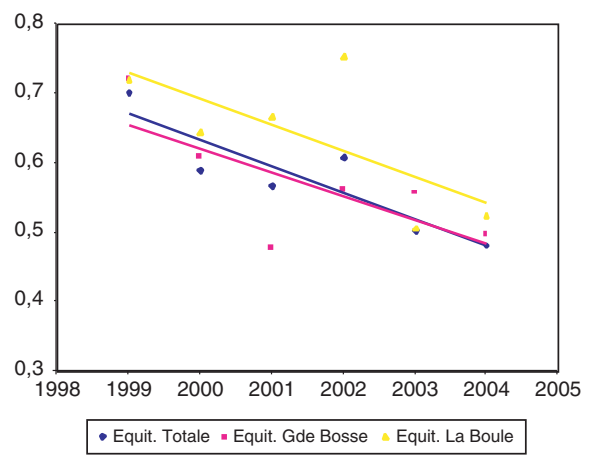

Fig. 5. Évolution de l'équitabilité aux deux stations de 1999 à 2004.

Fig. 5. Evolution of eveness in the two stations between 1999 and 2004.

entre les variations de température et celles des peuplements de jeunes poissons, sans tenir compte de l'effet spatial qui s'est avéré négligeable 
dans ce contexte pour comprendre l'évolution temporelle de la faune piscicole.

\subsection{Influence de la température sur les peuplements de jeunes poissons de l'année}

Le tableau des données de milieu comporte donc huit variables thermiques calculées pour chaque année et chaque station, soit 12 observations ( 6 années $\times 2$ stations). Le tableau faunistique comporte les mêmes 12 observations concernant 16 variables espèces. Ces deux tableaux sont soumis à une ACP avant couplage par analyse de co-inertie.

Un test de permutation permet d'estimer la validité de cette analyse. La valeur de co-inertie observée est comparée aux valeurs de coinertie calculées après 100 permutations aléatoires des lignes du tableau des données de milieu. Le test étant significatif ( $p<0,001)$, l'appariement des deux tableaux et la co-structure qui en découle ne sont pas liés au hasard.

En premier lieu, le graphe des valeurs propres de l'analyse de co-inertie (Fig. 6A) indique la prépondérance du premier axe de cette analyse $(76 \%$ de l'inertie totale) qui fera donc essentiellement l'objet des commentaires qui suivent.

La projection des axes issus des analyses séparées sur le plan formé par les axes de co-inertie démontre que les structures initiales de chacun des tableaux sont bien restituées dans la co-structure, en particulier sur le $1^{\mathrm{er}}$ axe (Fig. 6B). Ceci est confirmé par l'interprétation numérique de l'analyse de co-inertie (Tab. II) puisque les valeurs de variance issues de la co-inertie sont proches des valeurs d'inertie des analyses séparées (pour l'axe 1, 97\% et 92,8\% de l'inertie initiale sont conservés dans la co-inertie respectivement pour le tableau faunistique et le tableau des données de milieu). Enfin, la corrélation entre les coordonnées des observations, issues de la matrice espèces, et celles, issues de la matrice température est élevée, en particulier sur l'axe $1(r=0,879)$.

Les observations des années 2001 et 2003 se distinguent à la fois de par les caractéristiques thermiques et la composition faunistique : elles sont opposées selon l'axe 1, en particulier quand on considère la carte factorielle des observations dans le plan de coinertie selon les variables thermiques (Fig. 6D). Cette ordination contrastée semble liée aux extrêmes thermiques caractérisant à la fois l'année biologique dans son ensemble et la période de reproduction. En particulier, pour l'axe 1, ce sont les centiles 90 des températures de l'année biologique (T90biol) et les centiles 10 des températures de la période de reproduction (T10repro) qui semblent importants (Fig. 6C). La variabilité des températures durant les deux périodes considérées (année biologique et période de reproduction) (Cvbiol et Cvrepro) contribue dans une moindre mesure à l'axe 1. Ce gradient thermique entre les années 2001 et 2003 reflète effectivement les conditions climatiques caractéristiques de ces 2 années, l'année 2003 étant une année chaude et l'année 2001, une année fraîche. À ce gradient thermique correspond un gradient temporel moins 

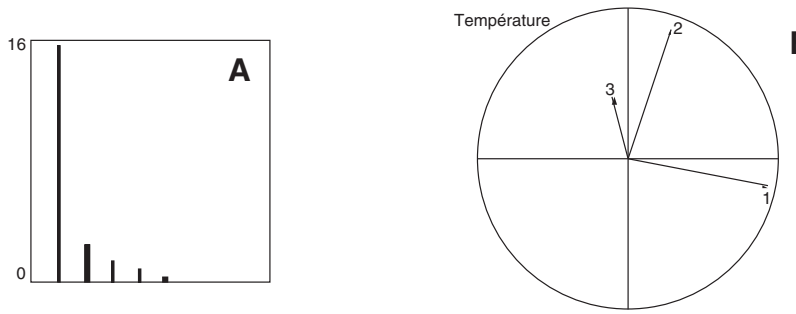

B
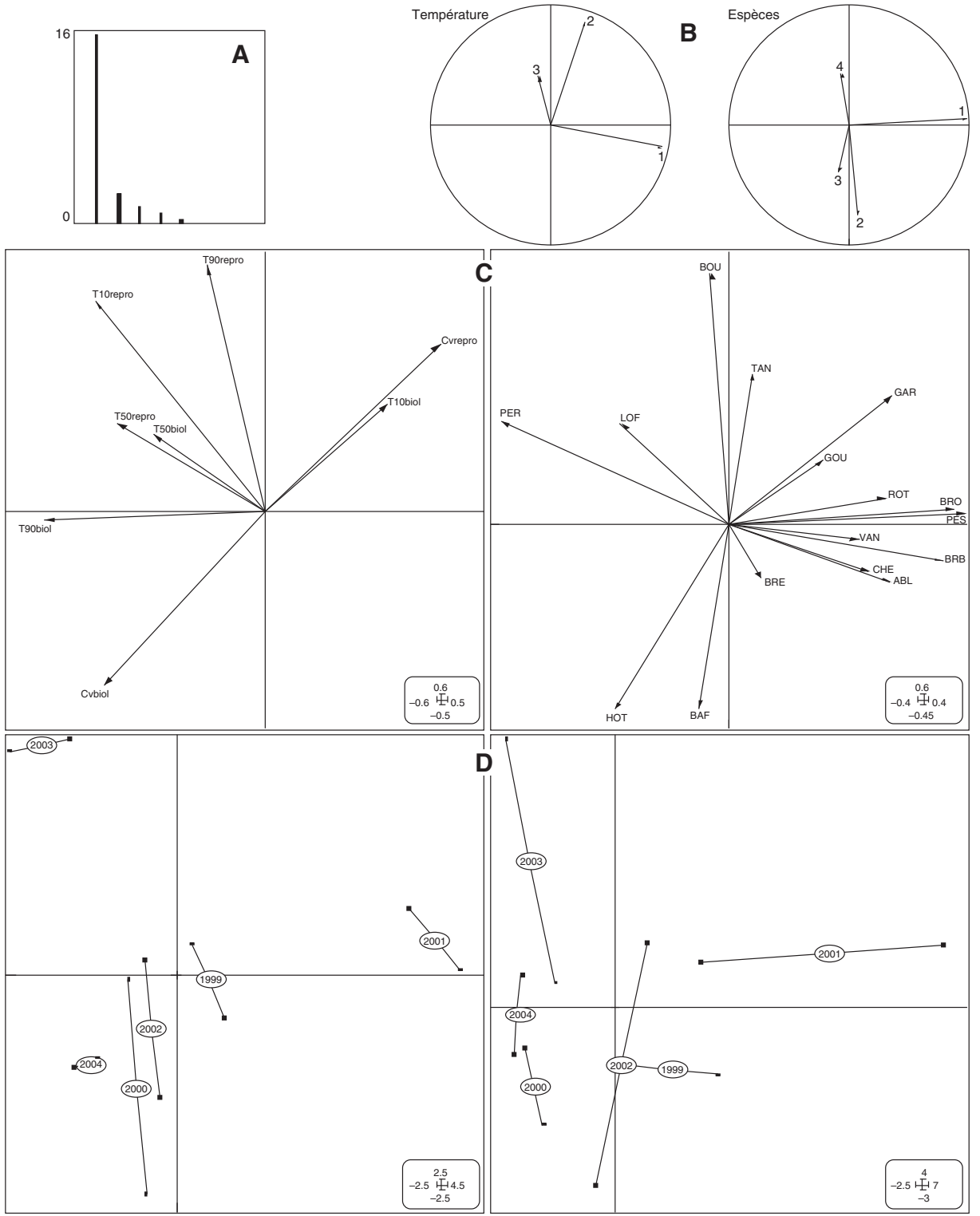

Fig. 6. Analyse de co-inertie. A) Histogramme des valeurs propres; B) Projections des axes d'inertie des analyses séparées sur le plan 1-2 de co-inertie; C) Cartes factorielles dans le plan 1-2 de coinertie des variables thermiques et des variables espèces (les codes figurent dans le tableau I); D) Cartes factorielles dans le plan 1-2 de co-inertie des observations regroupées par année.

Fig. 6. Co-inertia analysis. A) eigen values plot; B) projections of inertia axes from separated analyses onto the plan 1-2 of co-inertia; C) factorial maps of temperature variables and species variables in the plan 1-2 of co-inertia (species codes are in Table I); D) factorial maps of by-year observations in the plan 1-2 of co-inertia. 
Tableau II. Données numériques de l'analyse de co-inertie. Comparaison entre les inerties issues de l'analyse de co-inertie (variance) et des analyses séparées (inertie initiale).

Table II. Numerical data of co-inertia analysis. Comparison between inertia values resulting from co-inertia analysis (variance) and separated analyses (initial inertia).

\begin{tabular}{|l|c|c|c|c|c|c|}
\hline & Covariance & $\begin{array}{c}\text { Variance } \\
\text { température }\end{array}$ & $\begin{array}{c}\text { Variance } \\
\text { espèces }\end{array}$ & Corrélation & $\begin{array}{c}\text { Inertie initiale } \\
\text { température }\end{array}$ & $\begin{array}{c}\text { Inertie initiale } \\
\text { espèces }\end{array}$ \\
\hline Axe 1 & 3,954 & 3,527 & 5,742 & 0,879 & 3,801 & 5,896 \\
\hline Axe 2 & 1,574 & 1,750 & 2,510 & 0,751 & 1,817 & 3,126 \\
\hline
\end{tabular}

contrasté des années selon les espèces de poissons. Plusieurs espèces contribuent positivement à l'axe 1, telles que la perche soleil, le brochet, la brème bordelière ou encore le gardon, et semblent de fait, caractériser l'année 2001 (Fig. 6C). Plus globalement, la majorité des espèces de cyprinidés contribuent positivement à l'axe 1. Compte tenu du gradient thermique lié à cet axe, ce résultat est surprenant parce qu'il tend à suggérer que les plus fortes températures (à la fois en moyenne et en amplitude extrême) ne sont pas particulièrement favorables à leur recrutement. En revanche les espèces telles que la perche commune et la loche franche contribuent négativement à l'axe 1 (Fig. 6C) et semblent être caractéristiques des années biologiques les plus chaudes (2003 mais aussi 2004).

\subsection{Discussion}

Durant les 6 années de suivi, la richesse spécifique totale des peuplements de jeunes poissons de l'année a augmenté tandis que leur équitabilité a diminué. Au cours de la même période, les variations inter-annuelles de température se sont manifestées surtout par la variation des extrêmes thermiques hauts et bas ainsi que par l'amplitude des gammes de variation thermique tandis que les conditions thermiques moyennes des sites étudiés ont peu évolué.

L'étude montre par ailleurs que les variations inter-annuelles du recrutement répondent effectivement aux variables thermiques caractérisant les extrêmes (T90biol et T10repro principalement), les variables de tendance moyenne étant les moins significatives.

Les années « exceptionnelles » du point de vue hydroclimatique, que comporte le suivi (2001 et 2003) sont bien distinguées à la fois par les variables thermiques et les composantes faunistiques.

Un résultat singulier est que les années les plus chaudes semblent ne pas être associées à de fortes abondances de jeunes cyprinidés de l'année : classiquement, une température plus élevée (notamment sur la période «repro») devrait favoriser le recrutement des espèces de cyprinidés. Cette tendance a été observée notamment pour les peuplements de poissons du Haut-Rhône (Daufresne et al., 2003).

Ce résultat singulier pour la Seine est donc à confirmer en analysant non 
plus les données globalement mais en recherchant :

- l'effet thermie spécifique sur la taille des individus $0+$ : si la reproduction et la croissance des jeunes poissons sont effectivement accélérées lors des années plus chaudes, à date équivalente, les individus sont de plus grande taille, donc plus mobiles et éventuellement, la plupart des jeunes ont déjà changé d'habitats et in fine, quitté les milieux annexes tels que les deux stations étudiées. Ce phénomène justifierait qu'on observe des abondances moindres de jeunes cyprinidés justement lors des épisodes les plus chauds. Pour certaines espèces au contraire, il est possible que des températures exceptionnellement élevées aient un effet léthal vis-à-vis des jeunes stades (œuf puis embryon puis alevin), ce qui expliquerait de la même manière des abondances moindres.

- Les éventuelles interactions biotiques : la perche s'opposant aux autres espèces dans l'analyse globale, il existe peut être un effet prédateur-proie influençant les variations d'effectifs mais aussi les modes d'occupation des habitats. Notamment, des expérimentations ont mis en évidence que les jeunes gardons adaptaient leurs modes d'utilisation spatiale des habitats selon la présence des prédateurs que sont les perches (Eklov \& Persson, 1995). En revenant aux données initiales de capture par point, on peut rechercher un antagonisme éventuel dans l'occupation des habitats entre la perche et les jeunes $0+$ de cyprinidés.

\section{4 ÉVOLUTION À LONG TERME DES PEUPLEMENTS DE POISSONS SUR L'AXE SEINE (1990-2004)}

\subsection{Variations du peuplement de poissons}

Au total, 24 espèces ont été capturées à Choisy-le Roi contre 29 à Nogent-sur-Seine. Pour un effort de pêche comparable, 1056 individus ont été pêchés à Choisy-leRoi contre 1606 à Nogent-sur-Seine. Seules 16 espèces ont une occurrence strictement supérieure à deux à Choisy-le-Roi pour 23 espèces à Nogent-sur-Seine (Tab. III).

Le nombre d'espèces tend à augmenter dans les deux stations étudiées (Figs. 7 et 9) alors que l'indice de Shannon reste relativement stable. En revanche, les effectifs n'évoluent pas de la même façon aux deux stations. À Nogent-sur-Seine, ils sont relativement stables avec une chute notable en 1995 et 1996 et un pic en 2004 (Fig. 8) ; tandis qu'à Choisy-leRoi, ils semblent plus variables et sont les plus élevés en 1994, 1995 et 1997 (Fig. 10).

\section{2 Évolution des températures et des débits}

Les températures de l'eau à Choisyle-Roi sont plus élevées que celles de Nogent-sur-Seine : sur l'ensemble des années biologiques, donc de 1990 à 2004, la température moyenne de l'eau de Choisy-le-Roi est de $13,9^{\circ} \mathrm{C}$ pour $13,02^{\circ} \mathrm{C}$ à Nogent-surSeine (Figs. 11 et 12). Au printemps, la température moyenne de l'eau à Choisy-le-Roi est de $16,8^{\circ} \mathrm{C}$ 
Tableau III. Liste des espèces capturées avec leur occurrence respective dans les deux sites durant les 15 années étudiées.

Table III. List of caught species with their related occurrence in the two sites during the 15 study years.

\begin{tabular}{|c|c|c|c|c|c|}
\hline \begin{tabular}{|l|} 
Code \\
espèce
\end{tabular} & Nom vernaculaire & Nom scientifique & Famille & $\begin{array}{c}\text { Occurrence } \\
\text { à Choisy }\end{array}$ & $\begin{array}{c}\text { Occurrence } \\
\text { à Nogent }\end{array}$ \\
\hline $\mathrm{ABL}$ & Ablette & Alburnus alburnus & Cyprinidés & 14 & 15 \\
\hline ANG & Anguille & Anguilla anguilla & Anguillidés & 14 & 15 \\
\hline BAF & Barbeau Fluviatile & Barbus barbus & Cyprinidés & 1 & 0 \\
\hline $\mathrm{BOU}$ & Bouvière & Rhodeus sericeus $P$. & Cyprinidés & 2 & 15 \\
\hline BRB & Brème bordelière & Blicca bjoerkna $L$. & Cyprinidés & 4 & 14 \\
\hline BRE & Brème Commune & Abramis brama & Cyprinidés & 10 & 13 \\
\hline BRO & Brochet & Esox lucius L. & Esocidés & 10 & 15 \\
\hline CAS & Carassin & Carassius carassius $L$. & Cyprinidés & 1 & 1 \\
\hline $\mathrm{cco}$ & Carpe Commune & Cyprinus carpio L. & Cyprinidés & 2 & 3 \\
\hline $\mathrm{CHA}$ & Chabot & Cottus gobio L. & Cottidés & 3 & 3 \\
\hline $\mathrm{CHE}$ & Chevaine & Leuciscus cephalus $L$. & Cyprinidés & 15 & 15 \\
\hline EPI & Epinoche & Gasterosteus aculeatus $L$. & Gasterosteidés & 1 & 0 \\
\hline EPT & Epinochette & Pungitius pungitius $L$. & Gasterosteidés & 0 & 1 \\
\hline GAR & Gardon & Rutilus rutilus $L$. & Cyprinidés & 15 & 15 \\
\hline GOU & Goujon & Gobio gobio $L$. & Cyprinidés & 9 & 13 \\
\hline GRE & Grémille & Gymnocephalus cernua L. & Percidés & 0 & 5 \\
\hline HOT & Hotu & Chondrostoma nasus $L$. & Cyprinidés & 8 & 12 \\
\hline LOF & Loche Franche & Barbatula barbatula $L$. & Balitoridés & 0 & 8 \\
\hline LOR & Loche de Rivière & Cobitis taenia $L$. & Cobitidés & 0 & 6 \\
\hline LOT & Lote de Rivière & Lota lota & Lotidés & 1 & 3 \\
\hline LPP & Lamproie de Planer & Lampetra planeri $B$. & Pétromyzontidés & 0 & 2 \\
\hline $\mathrm{PCH}$ & Poisson Chat & Ictalurus melas $R$. & Ictaluridés & 4 & 3 \\
\hline PER & Perche & Perca fluviatilis $L$. & Percidés & 15 & 15 \\
\hline PES & Perche Soleil & Lepomis gibbosus $L$. & Centrarchidés & 9 & 13 \\
\hline PSR & Pseudorasbora & Pseudorasbora parva T.S. & Cyprinidés & 0 & 2 \\
\hline ROT & Rotengle & \multicolumn{2}{|c|}{ Scardinius erythrophthalmus L.Cyprinidés } & 14 & 15 \\
\hline SAN & Sandre & Stizostedion lucioperca L. & Percidés & 8 & 2 \\
\hline SPI & Spirlin & Alburnoides bipunctatus $B$. & Cyprinidés & 0 & 3 \\
\hline TAC & Truite Arc-en-Ciel & Oncorhynchus mykiss $W$. & Salmonidés & 1 & 0 \\
\hline TAN & Tanche & Tinca tinca $L$. & Cyprinidés & 4 & 14 \\
\hline VAI & Vairon & Phoxinus phoxinus $L$. & Cyprinidés & 0 & 2 \\
\hline VAN & Vandoise & Leuciscus leuciscus $L$. & Cyprinidés & 1 & 6 \\
\hline
\end{tabular}

pour $15,8^{\circ} \mathrm{C}$ à Nogent-sur-Seine, et en été, de $19,4^{\circ} \mathrm{C}$ à Choisy-le-Roi contre $17,9^{\circ} \mathrm{C}$ à Nogent-sur-Seine. Les moyennes des températures restent relativement stables, sauf lors des dernières années étudiées à
Nogent-sur-Seine, où l'on observe une augmentation des températures moyennes annuelle et estivale en 2003 et 2004, ainsi qu'un pic de la température moyenne printanière en 2003 (Fig. 11). À noter en 


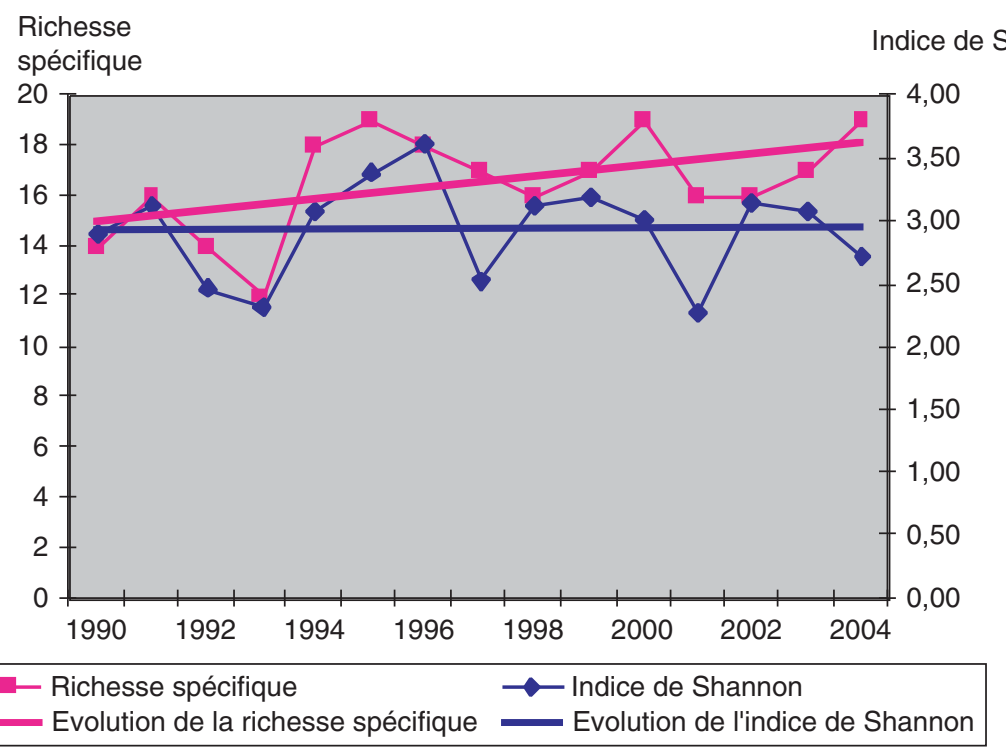

Fig. 7. Évolution du nombre d'espèces et de l'indice de Shannon à Nogent-sur-Seine entre 1990 et 2004.

Fig. 7. Evolution of species richness and Shannon's index at Nogent-sur-Seine between 1990 and 2004.

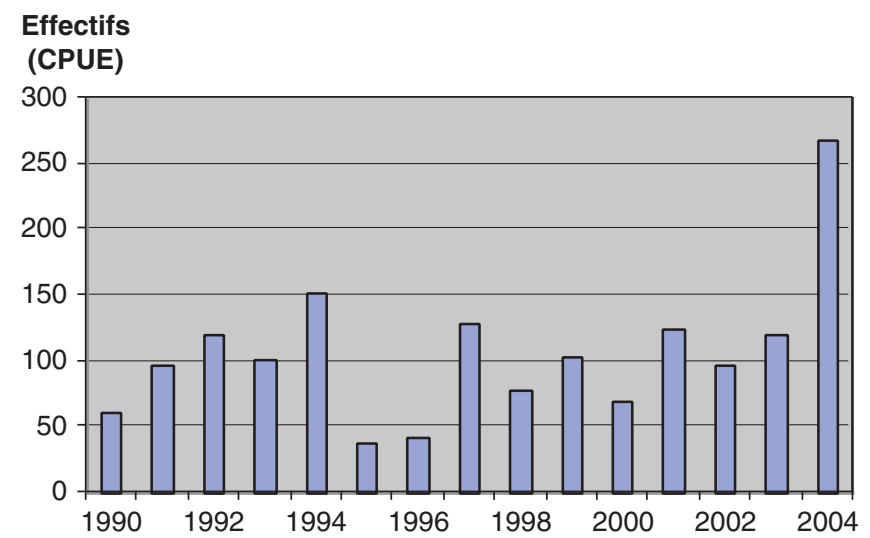

Fig. 8. Évolution des effectifs capturés à Nogent-sur-Seine entre 1990 et 2004.

Fig. 8. Evolution of caughts at Nogent-sur-Seine between 1990 and 2004.

particulier pour l'année biologique 2003 l'élévation de la température moyenne printanière qui dépasse la courbe des températures moyennes estivales. Ces particularités thermiques semblent atténuées sur le graphe des températures de Choisy-le-Roi (Fig. 12). 


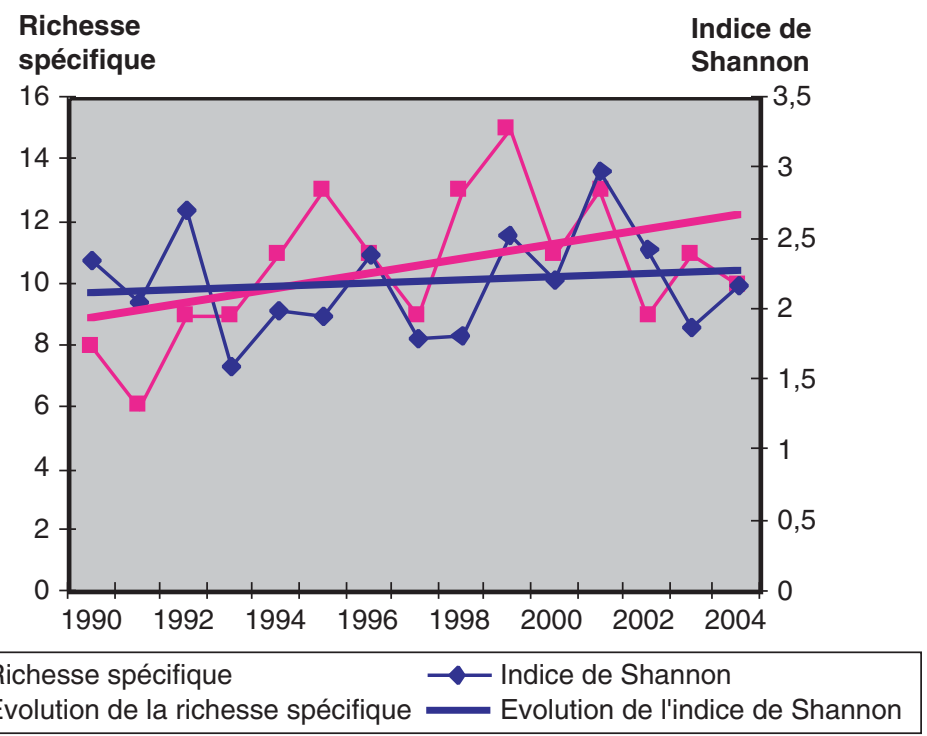

Fig. 9. Évolution du nombre d'espèces et de l'indice de Shannon à Choisy-le-Roi entre 1990 et 2004. Fig. 9. Evolution of species richness and Shannon's index at Choisy-le-Roi between 1990 and 2004.

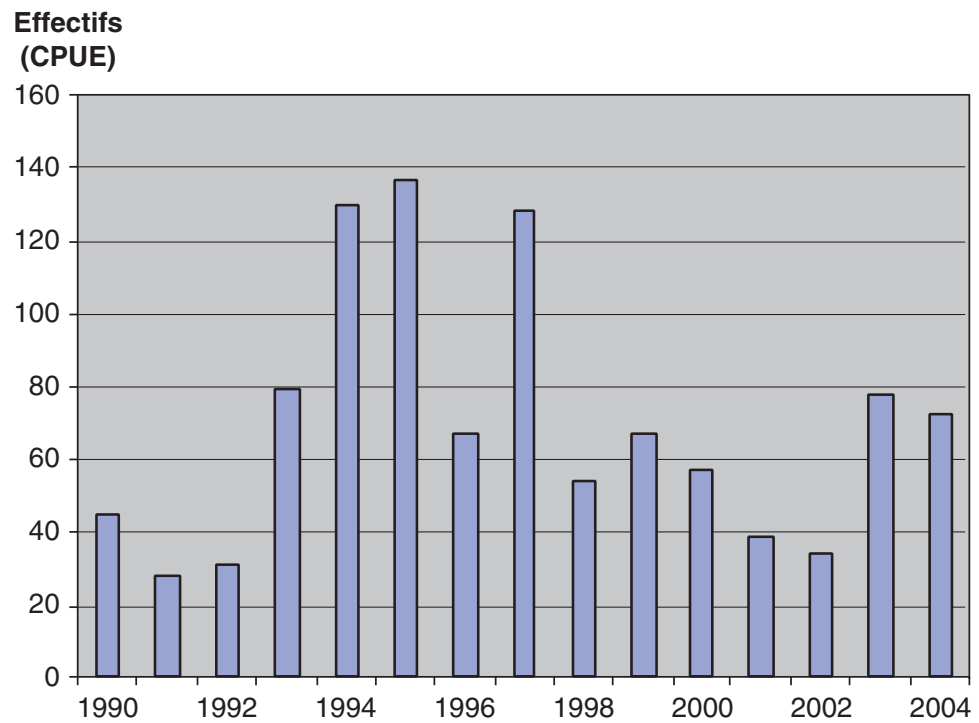

Fig. 10. Évolution des effectifs piscicoles capturés à Choisy-le-Roi entre 1990 et 2004.

Fig. 10. Evolution of caughts at Choisy-le-Roi between 1990 and 2004. 


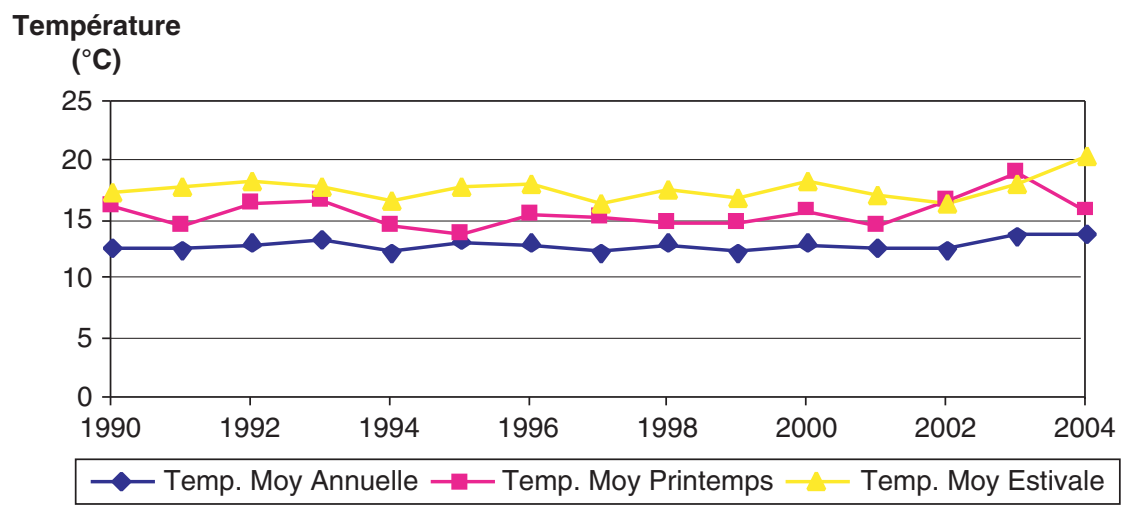

Fig. 11. Évolution des moyennes des températures de Nogent-sur-Seine de 1990 à 2004 aux trois périodes : du $1^{\mathrm{er}}$ avril au 30 juin (températures printanières), du $1^{\mathrm{er}}$ juillet au 31 octobre (températures estivales) et du $1^{\text {er }}$ juillet au 30 juin (températures annuelles).

Fig. 11. Evolution of mean water temperature at Nogent-sur-Seine between 1990 and 2004 for the three periods: from April 1st to June 30th (spring temperatures), from July 1st to October 31th (summer temperatures) and from July 1 st to June 30th (annual temperatures).

\section{Température}

$\left({ }^{\circ} \mathrm{C}\right)$

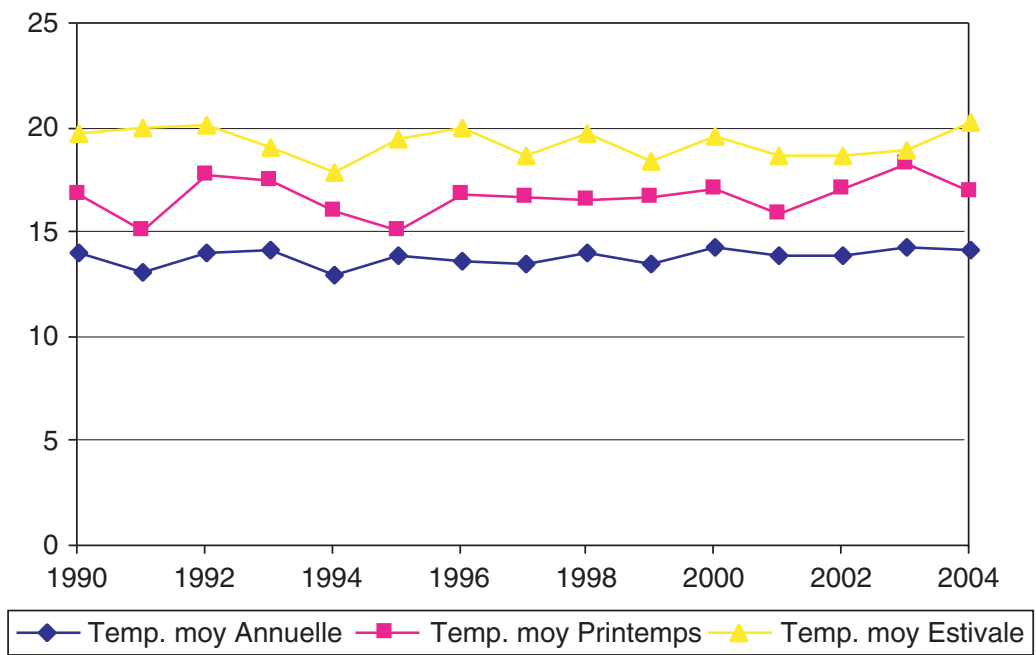

Fig. 12. Évolution des moyennes des températures de Choisy-le-Roi de 1990 à 2004 aux trois périodes : du $1^{\mathrm{er}}$ avril au 30 juin (températures printanières), du $1^{\mathrm{er}}$ juillet au 31 octobre (températures estivales), et du $1^{\mathrm{er}}$ juillet au 30 juin (températures annuelles).

Fig. 12. Evolution of mean water temperature at Choisy-le-Roi between 1990 and 2004 for the three periods: from April 1st to June 30th (spring temperatures), from July 1st to October 31th (summer temperatures) and from July 1 st to June 30 th (annual temperatures). 
Les débits sont plus élevés à Choisy-le-Roi qu'à Nogent-sur-Seine, du fait que Choisy-le-Roi se situe en aval de Nogent-sur-Seine et que la Seine a reçu entre temps des affluents majeurs, dont l'Yonne. La moyenne annuelle de débit est de 208009 L.s ${ }^{-1}$ à Choisy-le-Roi et de 76460 L.s $^{-1}$ à Nogent-sur-Seine. Au printemps, la moyenne est de 186274 L.s $^{-1}$ à Choisy-le-Roi et de 66466 L.S $^{-1}$ à Nogent-sur-Seine; en été, la moyenne est de 109776 L.s $^{-1}$ à Choisy-leRoi et de 49249 L.s $^{-1}$ à Nogent-surSeine.

Les graphes indiquant l'évolution des débits (Figs. 13 et 14) indiquent que le débit évolue de façon similaire aux deux stations. Quatre années sont particulièrement humides : 1994, 1995, 1998 et 2001, le maximum de débit étant atteint pour les deux stations en 2001. Ce sont les mêmes années aux deux stations. Les années sèches sont beaucoup plus nombreuses, en particulier de 1990 à 1993, 1996 et 1997.

Les évolutions comparées de la température de l'eau et du débit aux deux stations confirment que cellesci ont connu des conditions comparables au cours de la période étudiée, à l'ordre de grandeur près qui correspond au gradient longitudinal de taille du cours d'eau.

\subsection{Relations entre les peuplements de poissons et les conditions hydroclimatiques}

Au préalable, il est important de rechercher si les peuplements de poissons des deux sites évoluent de manière analogue au cours de la période d'étude. Une analyse de coinertie multiple (ACOM) est réalisée concernant les données piscicoles pour rechercher un tel phénomène de synchronie. L'ACOM est une

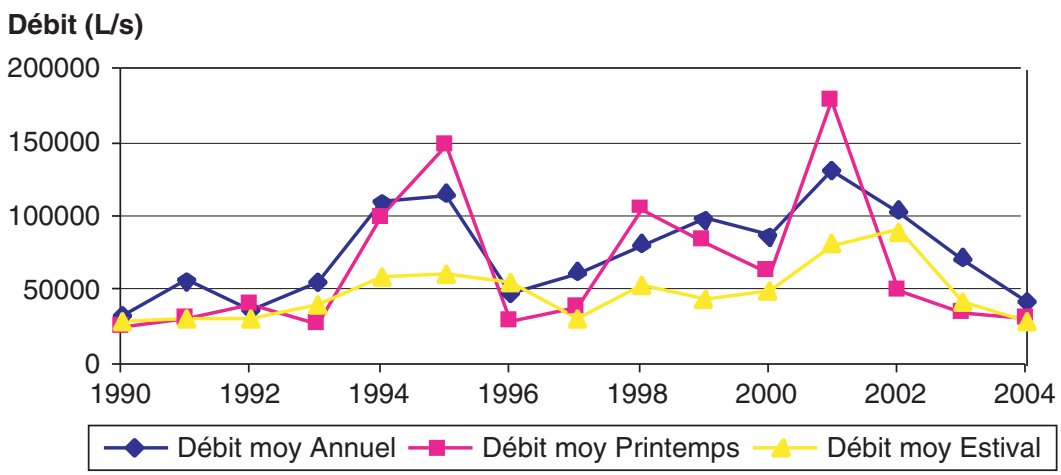

Fig. 13. Évolution des moyennes des débits de Nogent-sur-Seine de 1990 à 2004 aux trois périodes : $\mathrm{du} 1^{\mathrm{er}}$ avril au 30 juin (débit printanier), du $1^{\mathrm{er}}$ juillet au 31 octobre (débit estival) et du $1^{\mathrm{er}}$ juillet au 30 juin (débit annuel).

Fig. 13. Evolution of mean water discharge at Nogent-sur-Seine between 1990 and 2004 for the three periods: from April 1st to June 30th (spring discharge), from July 1st to October 31th (summer discharge) and from July 1st to June 30th (annual discharge). 


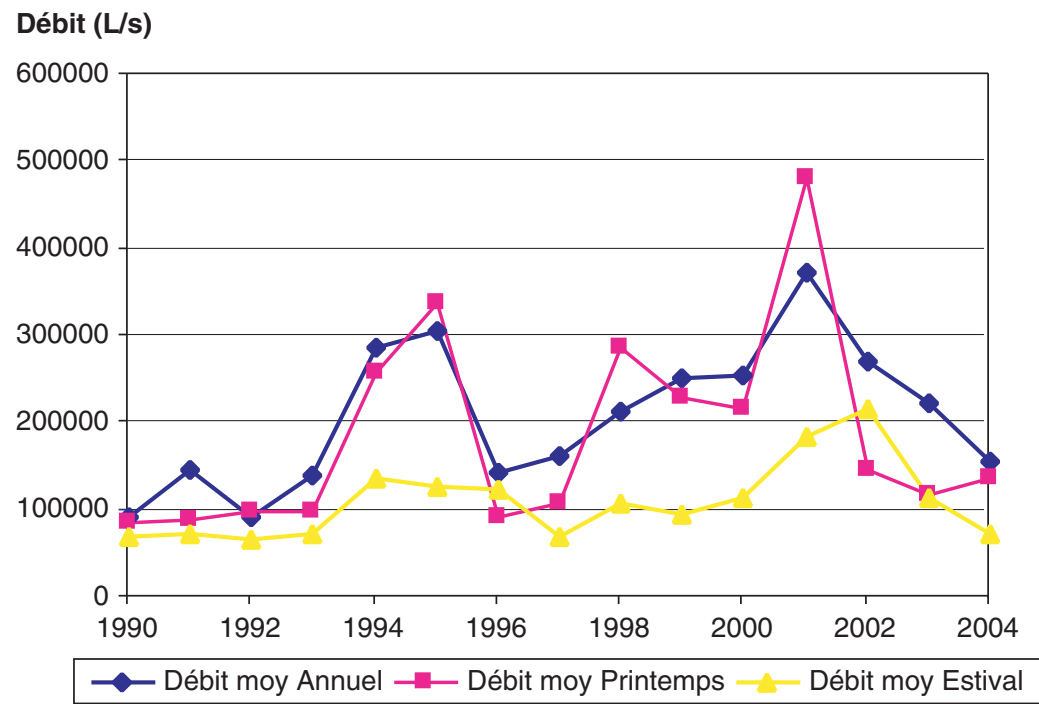

Fig. 14. Évolution des moyennes des débits de Choisy-le-Roi de 1990 à 2004 aux trois périodes : du $1^{\mathrm{er}}$ avril au 30 juin (débit printanier), du $1^{\mathrm{er}}$ juillet au 31 octobre (débit estival), du $1^{\mathrm{er}}$ juillet au 30 juin (débit annuel).

Fig. 14. Evolution of mean discharge at Choisy-le-Roi between 1990 and 2004 for the three periods: from April 1st to June 30th (spring discharge), from July 1st to October 31th (summer discharge) and from July 1 st to June 30th (annual discharge).

analyse multi-tableaux : c'est une méthode d'ordination basée sur le critère d'optimisation de la covariance entre tableaux. Cette méthode s'avère adéquate pour rechercher la synchronie des communautés d'organismes aquatiques de plusieurs sites d'étude (Bady et al., 2004). Cette analyse a permis de vérifier qu'une évolution temporelle des peuplements de poissons, commune aux deux sites, existe. II est donc légitime de rechercher l'effet de facteurs abiotiques tels que les conditions hydroclimatiques expliquant cette synchronie.

Une analyse de co-inertie réalisée à partir du couplage du tableau faunistique (30 observations année-site $\times 24$ espèces) et du tableau mésologique
(30 observations année-site $\times 6$ variables hydroclimatiques) analysés au préalable par une ACP normée permet de dégager leur co-structure. Les données des deux sites étant regroupées au sein d'un seul tableau, le tableau faunistique comporte 24 espèces résultant du cumul des espèces des deux sites.

Un test de permutation réalisé au préalable permet de vérifier que cette co-structure n'est pas aléatoire. À l'issue de 100 permutations aléatoires, l'inertie observée dans l'analyse de co-inertie (inertie $=12,162$ ) est très supérieure aux valeurs d'inertie obtenues par simulation $(p<0,001)$, indiquant que l'analyse de co-inertie est valide. 
La corrélation globale entre les deux tableaux est de 0,42 alors que les corrélations entre les deux tableaux sur les deux premiers axes de co-inertie (82\% et $65 \%$ respectivement) sont élevées, ce qui indique une bonne correspondance de leurs structures. Cette information est confirmée par la représentation graphique des projections des axes d'inertie des ACP initiales dans le premier plan de coinertie (Fig. 15D).

Les relations entre les variables mésologiques et les espèces sont ensuite appréciées par l'interprétation des cartes factorielles. II existe apparemment un gradient hydroclimatique selon le premier axe de l'analyse de co-inertie auquel l'ensemble des variables de température et de débit est fortement corrélé négativement (Fig. 15B). Le $2^{\mathrm{e}}$ axe matérialise l'opposition attendue entre valeurs de débit et de température. À ce gradient de milieu répond une évolution des espèces, la plupart étant opposées essentiellement à la perche (PER) et au sandre (SAN) selon le premier axe de l'analyse (Fig. 15A). Autrement dit, il résulte de cette première interprétation l'observation suivante : les deux espèces, perche et sandre, sont plutôt associées aux fortes valeurs des variables de température et débit.

La représentation des projections des observations (Fig. 15C) indique qu'en fait le gradient mis en évidence correspond à un effet spatial puisque tous les relevés de la station de Nogent-sur-Seine (de 1 à 15) occupent la partie droite du plan alors que ceux de la station de Choisyle-Roi (de 16 à 30) se situent dans la partie gauche. En effet, la station de Choisy-le-Roi se caractérise par des débits et des températures plus élevées du fait de sa situation plus en aval de la Seine relativement à la station de Nogent-sur-Seine. Par conséquent, l'association précédemment notée entre les espèces perche et sandre et les fortes valeurs des variables de température et débit est liée à des différences entre les deux sites et non pas à une évolution temporelle particulière des conditions hydroclimatiques.

Pour s'affranchir de cet effet spatial, le même type d'analyse a été mis en œuvre pour chaque station séparément, mais sans résultat parce qu'aucune des analyses n'est alors valide, tel que l'indique le test de permutation non significatif. Par conséquent, on ne peut pas conclure à un effet significatif des conditions hydroclimatiques sur le peuplement de poissons dans chacun des deux sites considéré séparément.

\subsection{Discussion}

Durant les 15 années de suivi, la richesse spécifique des peuplements de poissons a augmenté dans les deux stations étudiées, alors que la diversité de Shannon est demeurée stable. L'évolution des températures moyennes calculées pour les périodes annuelles et saisonnières (printemps et été) indique une certaine stabilité. Toutefois, la température printanière de 2003 et la température estivale de 2004, particulièrement élevées, se singularisent à la station de Nogent-surSeine. En revanche, l'évolution des débits est entièrement similaire aux deux stations, avec, pour la période étudiée, un maximum enregistré en 2001.

Malgré des différences de composition faunistique, la synchronie 


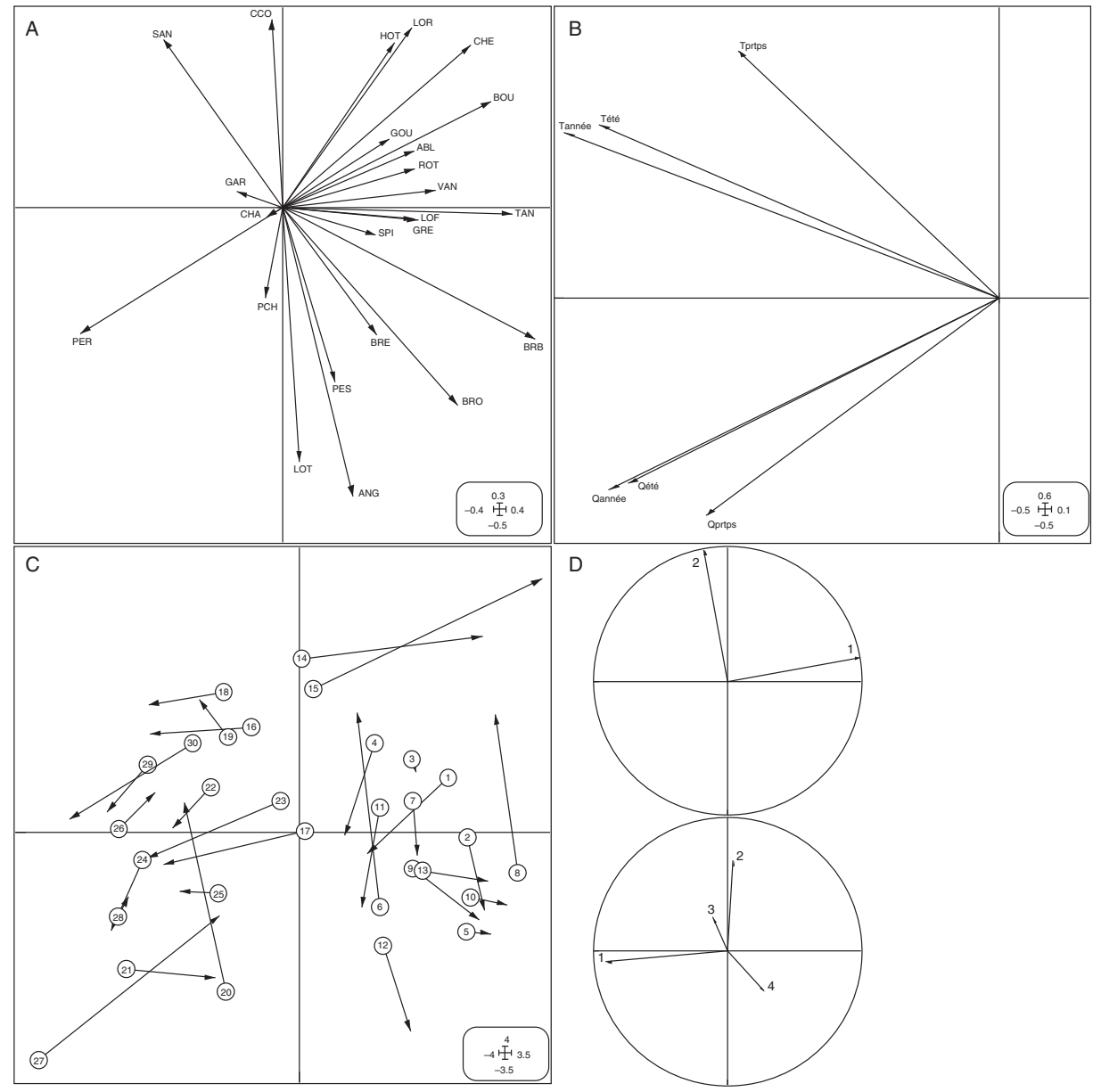

Fig. 15. Analyse de co-inertie. A) Carte factorielle des espèces dans le plan 1-2 de co-inertie. B) Carte factorielle des variables hydroclimatiques dans le plan 1-2 de co-inertie. C) Projection des observations année-site dans le plan 1-2 de co-inertie : le cercle correspond à la position de l'observation correspondant aux variables hydroclimatiques tandis que l'extrémité de la flèche indique sa position relative aux variables faunistiques (de 1 à 15 : observations de Nogent; de 16 à 30 : observations de Choisy). D) Projections des axes des ACP des tableaux initiaux dans le plan 1-2 de co-inertie (en haut : milieu; en bas : espèces).

Fig. 15. Co-inertia analysis. A) factorial map of species in the plan 1-2 of co-inertia; B) factorial map of hydroclimatic variables in the plan 1-2 of co-inertia; $\mathrm{C}$ ) projection of by year-sites observations in the plan 1-2 of co-inertia: circles indicate observation location due to hydroclimatic variables whereas arrows extremity indicates observation location due to faunistic variables (from 1 to 15 : observations from Nogent; from 16 to 30: observations from Choisy); D) projections of axes from separated analyses (PCA) onto the plan 1-2 of co-inertia (at the top: environmental data; at the down: fish data). 
des peuplements de poissons des deux stations est avérée. Comme par ailleurs, les conditions hydroclimatiques évoluent de manière similaire aux deux stations, on pouvait s'attendre à une forte corrélation entre les peuplements de poissons et les variables hydroclimatiques aux deux stations, ce qui n'est pas le cas. L'effet spatial entre stations est prépondérant dans les analyses. Néanmoins, on peut difficilement justifier la synchronie des peuplements aux deux stations autrement que par l'effet des conditions climatiques, les autres facteurs pouvant expliquer la synchronie étant peu probables dans ce contexte. Les fluctuations synchrones de populations géographiquement espacées sont généralement expliquées par des événements climatiques ou des échanges d'individus par processus de dispersion entre différentes populations (Grenouillet et al., 2001).

La dispersion d'individus entre les deux stations est peu réaliste car celles-ci sont distantes de près de 150 km, ce qui dépasse largement la taille du domaine vital des espèces qui composent les peuplements des deux stations. Cette remarque nous amène à proposer deux explications alternatives :

- la synchronie est effectivement due aux variations hydroclimatiques mais les variables retenues ne sont pas les plus pertinentes: il faudrait caractériser les variations hydroclimatiques par les extrêmes et non pas par les moyennes;

- II n'est peut être pas suffisant de ne retenir que les conditions hydroclimatiques de l'année qui précède l'échantillonnage : en effet, comme les campagnes d'échantillonnage ont lieu au printemps, la composante «juvénile de l'année » du peuplement n'est pas présente dans les captures alors que c'est elle qui est le plus susceptible de répondre «instantanément » aux variations des conditions hydroclimatiques régnant au cours de l'année écoulée. II y a peut être un effet retard à prendre en compte pour un peuplement de poissons composé essentiellement d'individus adultes, au moins pour les Cyprinidés qui constituent l'essentiel du peuplement.

Par rapport au travail antérieur de Daufresne (2005), sur le lien thermiepeuplement de poissons à Nogentsur-Seine, l'effet de la variabilité hydroclimatique n'est pas si évident. II est vrai qu'au lieu de corréler directement les variables hydroclimatiques au peuplement de poissons, il recherchait plutôt les effets potentiels d'une augmentation progressive de la température (en l'absence de données hydrométriques) sur les composantes des peuplements de poissons. Un effet attendu est notamment la progression d'espèces sudistes thermophiles au détriment des espèces nordiques et sténothermes d'eaux froides au sein des peuplements.

Pour mémoire, son travail mettait notamment en évidence une corrélation significative entre la température moyenne de reproduction et l'évolution du pourcentage des espèces sudistes et thermophiles au sein du peuplement de poissons, en cohérence avec les prédictions des conséquences du réchauffement climatique. Pour la station de Nogent-sur-Seine cependant, 
l'évolution était marquée concernant le trait latitudinal des espèces (sudiste vs. nordique) mais atténuée concernant leurs préférences thermiques (thermophile vs. sténotherme d'eau froide).

\section{CONCLUSION GÉNÉRALE}

Globalement les peuplements de poissons de la Seine ne manifestent pas de réponse biologique aux conditions moyennes thermiques. Chez les jeunes poissons de l'année, les variables thermiques extrêmes sont prépondérantes par rapport aux conditions moyennes. Concernant les peuplements adultes, nous n'avons pas réussi à mettre en évidence un effet direct des variables hydroclimatiques moyennes. La difficulté à laquelle on se heurte est d'identifier les variables climatiques pertinentes qui vont être corrélées à des réponses biologiques au niveau des peuplements de poissons. II est possible que la tendance moyenne d'augmentation de la température ne soit pas suffisamment contrastée, ou que notre série chronologique ne soit pas suffisamment longue pour dégager des réponses biologiques nettes.

II ne faut pas non plus négliger le fait que la Seine, par rapport au Rhône par exemple, est un fleuve de plaine, à régime pluvial. Ces conditions naturelles font sans doute que le fond faunistique est naturellement composé d'espèces (ou d'individus) à plus forte tolérance thermique, ce qui expliquerait pour l'instant une atténuation des effets potentiels de l'évolution des conditions hydroclimatiques sur les peuplements de poissons de la Seine. II est bien connu que l'effet des variations de température sur les espèces de poissons dépend dans une certaine mesure des conditions d'acclimatation.

Enfin, l'étude du recrutement suggère qu'il pourrait $y$ avoir un effet important de la précocité des saisons (des températures plus douces observées plus tôt au printemps) sur les variations d'abondance des jeunes poissons de l'année. Notamment, cet effet "précocité » pourrait influencer les interactions biotiques (typiquement les relations prédateurs proies) et modifier alors les équilibres entre espèces.

\section{REMERCIEMENTS}

Les auteurs remercient respectivement Nils Fauchon (Veolia) et JeanMaxence Ditche (ONEMA) pour la mise à disposition des données de température et des données piscicoles à la station de Choisy-le-Roi. Ils remercient également Alain Poirel (EDF) et Roxane Frichet pour leur travail préliminaire sur les données utilisées dans cette étude.

\section{RÉFÉRENCES BIBLIOGRAPHIQUES}

Bady P., Dolédec S., Dumont B. \& Fruget J.-F., 2004. Multiple co-inertia analysis: a tool for assessing synchrony in the temporal variability of aquatic communities. C.R. Biologies 327 : 29-36.

Cattaneo, F., Lamouroux N., Breil P. \& Capra H., 2002. The influence of hydrological and biotic processes on brown trout (Salmo trutta) population dynamics. Can. J. Fish. Aquat. Sci. 59 (1) : 12-22.

Caudy O., 2004. Variabilité temporelle des peuplements ichtyologiques d'un bras 
mort de la Seine (1999-2003). Mémoire de DESS GIREC, Université de Metz, $59 \mathrm{p}$.

Daufresne M., Roger M-C., Capra H. \& Lamouroux N., 2003. Long term changes within the invertebrate and fish communities of the Upper Rhône River: effects of climatic factors. Global Change Biology 10 : 124-140.

Daufresne M. \& Boët P., 2005. Évolution temporelle de la structure des peuplements piscicoles de grands fleuves français. Rapport d'études EDF, 19 p.

Dolédec S. \& Chessel D., 1994. Coinertia analysis: an alternative method for studying species-environment relationships. Freshwater Biology 31 : 277-294.

Eklov P. \& Persson L., 1995. Speciesspecific antipredator capacities and prey refuges : Interactions between piscivorous perch (Perca fluviatilis) and juvenile perch and roach (Rutilus rutilus). Behav. Ecol. Sociobiol. 37 (3) : 169-178.

Grenouillet G., Hugueny B., Carrel G., Olivier J-M. \& Pont D., 2001. Largescale synchrony and inter-annual variability in roach recruitment in the Rhône River : the relative role of climatic factors and density-dependent processes. Freshwater Biology 46 : 11-26. 